\title{
Longitudinal dynamics of high gain free electron laser amplifiers
}

\author{
G. Dattoli* and E. Sabia ${ }^{\dagger}$ \\ ENEA-Centro Ricerche Frascati, via E. Fermi, 45, IT 00044 Frascati, Rome, Italy
}

\section{P. L. Ottaviani}

INFN-Sezione di Bologna, Viale B. Pichat, 6/2, IT 40127 Bologna, Italy

S. Pagnutti ${ }^{\S}$

ENEA-Centro Ricerche Bologna, via Martiri di Monte Sole, 4, IT 40129 Bologna, Italy

V. Petrillo"

\begin{abstract}
Universita' degli Studi di Milano, via Celoria 16, IT 20133 Milano, Italy, and INFN-Mi, via Celoria 16, IT 20133 Milano, Italy
(Received 29 October 2012; published 12 March 2013)

We consider the dynamics of a coherent seed undergoing a free electron laser high gain amplification. We discuss the dispersion of the optical packet during the interaction and study the problem using different formalisms, including that of the Wigner distribution. The analysis we develop is mainly based on a 1D procedure; the inclusion of transverse longitudinal interplay is also briefly discussed.
\end{abstract}

DOI: 10.1103/PhysRevSTAB.16.030704

\section{INTRODUCTION}

The free electron laser (FEL) high gain equation in the small signal approximation, with the inclusion of pulse propagation effects, is written [1]

$$
\begin{aligned}
\partial_{\tau} a(z, \tau) & =i \pi g_{0}(z+\Delta \tau) \int_{0}^{\tau} \tau^{\prime} e^{-i \nu \tau^{\prime}} a\left(z+\Delta \tau, \tau-\tau^{\prime}\right) d \tau^{\prime}, \\
\Delta & =N \lambda, \quad \nu=2 \pi N \frac{\omega_{0}-\omega_{s}}{\omega_{0}}, \quad \tau \simeq \frac{c t}{L_{u}}, \\
L_{u} & =N \lambda_{u}, \quad \omega_{0}=\frac{2 \pi c}{\lambda_{0}}=c k_{0}, \quad \lambda_{0}=\frac{\lambda_{u}}{2 \gamma^{2}}\left(1+\frac{K^{2}}{2}\right)
\end{aligned}
$$

with $a(z, \tau)$ being the Colson's dimensionless amplitude [2]. The equation is formulated using the $z$ frame, associated with the optical packet distribution, and the $\tau$ variable, which is essentially the propagation coordinate along the undulator. The electron bunch, whose longitudinal shape has been included in the small signal gain coefficient, is assumed to be fixed. The optical field slips over the electrons and experiences an interaction responsible for a dispersive behavior yielding, among other things, a reduction of the group velocity of the radiation.

\footnotetext{
*dattoli@frascati.enea.it

†sabia@frascati.enea.it

${ }^{\ddagger}$ pl.ottaviani@alice.it

pagnutti@bologna.enea.it

"vittoria.petrillo@mi.infn.it
}

Published by the American Physical Society under the terms of the Creative Commons Attribution 3.0 License. Further distribution of this work must maintain attribution to the author(s) and the published article's title, journal citation, and DOI.
PACS numbers: 41.60.Cr, 52.59.Rz, 02.60.Nm, 02.30.Vv

In the following we will consider the amplification of a coherent seed packet and define the detuning $\nu$ with respect to the input seed frequency. If we assume that the electron bunch is significantly larger than the packet width, the small signal gain coefficient $g_{0}=\frac{(4 \pi N \rho)^{3}}{\pi}$, where $\rho$ is the Pierce parameter introduced by Bonifacio et al. in Ref. [3], can be assumed independent of the electron bunch longitudinal profile.

The use of the identity

$$
e^{\alpha \partial_{z}} f(z)=f(z+\alpha)
$$

allows one to formally eliminate the residual dependence on the $z$ variable by including the effect of the translation, due to the slippage $\Delta$, in the detuning parameter, thus rewriting Eq. (1.1) in the form

$$
\begin{aligned}
\partial_{\tau} a(z, \tau) & =i \pi g_{0} \int_{0}^{\tau} \tau^{\prime} e^{-i \hat{\nu} \tau^{\prime}} a\left(z, \tau-\tau^{\prime}\right) d \tau \\
\hat{\nu} & =\nu+i \Delta \partial_{z}
\end{aligned}
$$

Equation (1.3) is now formally equivalent to the 1D FEL high gain equation without pulse propagation dynamics. It can be therefore solved using the Volterra series method involving the gain approximants expansion proposed in Ref. [4]. The presence of the shift operator in the detuning parameter provides the interpretation of the approximants as evolution operators acting on the input seed amplitude.

Following Ref. [4], we write the relevant solution as

$$
\begin{aligned}
a(z, \tau) & =\hat{U}(\tau) a_{0}(z), \\
\hat{U}(\tau) & =\left[\hat{1}+\sum_{n=1}^{\infty}\left(i \pi g_{0}\right)^{n} g_{n}(\hat{\nu}, \tau)\right],
\end{aligned}
$$


where $\hat{U}(\tau)$ is the evolution operator acting on the initial seed amplitude

$$
a_{0}(z)=\frac{A_{0}}{\sqrt[4]{2 \pi \sigma^{2}}} e^{-z^{2} / 4 \sigma^{2}}
$$

and $g_{n}(\hat{\nu}, \tau)$ is the gain approximant evolution operator provided by

$$
\begin{aligned}
g_{n}(\hat{\nu}, \tau) & =M_{n} \tau^{3 n} \exp \left[-\frac{i}{2} \frac{\lambda_{n} \tau}{\gamma_{n}^{2}} \hat{\nu}-\frac{1}{2} \frac{\tau^{2}}{\gamma_{n}^{2}} \hat{\nu}^{2}\right], \\
M_{n} & =\frac{1}{(3 n) !}, \quad \gamma_{n}=(3 n+1) \sqrt{\frac{3 n+2}{2 n(n+1)}} \\
\lambda_{n} & =2(3 n+1) \frac{3 n+2}{n+1} .
\end{aligned}
$$

The optical field can therefore be expanded in gain amplitudes $a_{n}(z, \tau)$ according to the identity

$$
a(z, \tau)=\sum_{n=0}^{\infty}\left(\pi g_{0}\right)^{n} a_{n}(z, \tau), \quad a_{0}(z, \tau)=a_{0}(z)
$$

and for $n>0$

$$
\begin{aligned}
a_{n}(z, \tau)= & i^{n} g_{n}(\hat{\nu}, \tau) a_{0}(z) \\
= & i^{n} M_{n} \tau^{3 n} e^{-(1 / 2)\left(\nu \tau / \gamma_{n}^{2}\right)\left(i \lambda_{n}+\nu \tau\right)} \\
& \times e^{\left(\Delta \tau / 2 \gamma_{n}^{2}\right)\left(\lambda_{n}-2 i \nu \tau\right) \partial_{z}+\left(\tau^{2} / 2 \gamma_{n}^{2}\right) \Delta^{2} \partial_{z}^{2}} a_{0}(z) .
\end{aligned}
$$

The explicit form of the evolution of the input seed is obtained by specifying the effect of the exponential containing the derivative operators on $a_{0}(z)$.

By recalling Eq. (1.3) and [5],

$$
e^{\beta \partial_{z}^{2}} e^{-z^{2}}=\frac{1}{\sqrt{1+4 \beta}} e^{-\left(z^{2} / 1+4 \beta\right)},
$$

we eventually end up with, for $n>0$,

$$
\begin{aligned}
a_{n}(z, \tau)= & \frac{A_{n}(\nu, \tau)}{\sqrt[4]{2 \pi \sigma_{n}^{2}(\tau)}} e^{i k_{n}\left[z+\zeta_{n}(\tau)\right]-i \omega_{n} \tau+i n(\pi / 2)} \\
& \times e^{-\left[\left(z+\zeta_{n}(\tau)\right]^{2} /\left[4 \sigma_{n}^{2}(\tau)\right]\right.} \\
A_{n}(\nu, \tau)= & A_{0} M_{n} \tau^{3 n} \sqrt{\frac{\sigma}{\sigma_{n}(\tau)}} e^{-1 / 2\left(\nu \tau / \gamma_{n}\right)^{2}\left\{1-1 / 2 \gamma_{n}^{2}\left[\Delta \tau / \sigma_{n}(\tau)\right]^{2}\right\}}, \\
\sigma_{n}(\tau)= & \sigma \sqrt{1+\frac{1}{2}\left(\frac{\Delta \tau}{\gamma_{n} \sigma}\right)^{2}}, \quad \zeta_{n}(\tau)=\frac{1}{2} \frac{\lambda_{n} \Delta \tau}{\gamma_{n}^{2}}, \\
k_{n}(\nu, \tau)= & \frac{\nu \Delta}{2 \gamma_{n}^{2}}\left(\frac{\tau}{\sigma_{n}(\tau)}\right)^{2}, \quad \omega_{n}(\nu)=\frac{1}{2} \frac{\lambda_{n} \nu}{\gamma_{n}^{2}} .
\end{aligned}
$$

Each partial amplitude is interpreted as an optical packet having a shifted centroid, with respect to the original seed packet.

In this paper we will discuss the evolution of an input seed with particular reference to its spectral and spatial widths. We will analyze the mechanism of packet velocity reduction and introduce a suitable FEL refractive index.
We will exploit different techniques, including the evolution of the Wigner distribution [6] associated with the amplified field.

\section{SOME DETAILS ON THE OPTICAL PACKET EVOLUTION}

Equation (1.1) implies that the optical field evolution should be interpreted with respect to the $z$ frame, which moves rigidly at the light velocity. The portrait of the optical profile in $z$, taken at a given position along the undulator, provides various information including, e.g., those relevant to the group velocity.

According to the previous discussion, the centroids of the gain amplitude packets shift back, during the interaction with the e-beam, to the position $z_{n}=-\zeta_{n}(\tau)$. This effect can be interpreted as a manifestation of the fact that the group velocity of the optical packet has been slowed down by the FEL interaction itself.

According to Eqs. (1.6) and (1.9), we find that the shift back position of the optical packet is

$$
\zeta_{n}(\tau)=\frac{1}{2} \frac{\lambda_{n} \Delta \tau}{\gamma_{n}^{2}}=\frac{2 n}{3 n+1} \Delta \tau
$$

Since $\zeta_{n}(\tau)$ measures a relative position with respect to the $z$ frame assumed to move at the velocity of light, we argue that the packet group velocity is less than the light velocity and that it is given by

$$
v_{g, n}=c-\frac{d}{d t} \zeta_{n}(\tau)=c\left(1-\frac{2 n}{3 n+1} \frac{\lambda}{\lambda_{u}}\right) .
$$

In the high gain limit $(n \gg 1)$, we find

$$
v_{g} \simeq c\left(1-\frac{2}{3} \frac{\lambda}{\lambda_{u}}\right)
$$

Furthermore, since the longitudinal velocity of the electrons is

$$
v_{z} \simeq c\left(1-\frac{\lambda}{\lambda_{u}}\right),
$$

we obtain that, after one undulator passage, the difference between the optical packet and electron bunch centroids, measured in terms of slippage length, is

$$
\tilde{\Delta} \simeq \frac{\Delta}{3} .
$$

From Eq. (2.3) it also follows that the group velocity (in the high gain limit) can be cast in the form (see Bonifacio et al.) [7]

$$
v_{g} \simeq \frac{\omega}{k+\frac{2}{3} k_{u}}
$$

while in the low gain limit $(n=1)$ the same procedure yields 


$$
v_{g} \simeq \frac{\omega}{k+\frac{1}{2} k_{u}} .
$$

In the low gain regime the group velocity is slightly larger than the high gain limit since the refractive index associated with the interaction is lower.

The procedure we have outlined allows some analytical progress in understanding the high gain pulse evolution. If we assume that we operate the amplification process at zero detuning $(\nu=0$, the general case is discussed in the Appendix), the problem of studying the optical packet evolution is greatly simplified and indeed from Eq. (1.9) we find (we have omitted the dependence on $\nu, \tau$ in the $A$, $\sigma_{n}, \zeta_{n}$ functions to deal with more manageable expressions)

$$
a_{n}(z, \tau)=\frac{A_{n}}{\sqrt[4]{2 \pi \sigma_{n}^{2}}} e^{i n(\pi / 2)} e^{-\left(z+\zeta_{n}\right)^{2} / 4 \sigma_{n}^{2}}
$$

The intensity growth is obtained by integrating the total field amplitude on the packet coordinate $z$, thus getting ${ }^{1}$

$$
\begin{aligned}
I(\tau)= & |a(\tau)|^{2}=\int_{-\infty}^{+\infty}|a(z, \tau)|^{2} d z \\
= & \sqrt{2} \sum_{m=0}^{\infty} \sum_{n=0}^{\infty}\left(\pi g_{0}\right)^{n+m} A_{n} A_{m}^{\star} \sqrt{\frac{\sigma_{n} \sigma_{m}}{\sigma_{n}^{2}+\sigma_{m}^{2}}} \\
& \times e^{\alpha_{n m}} \cos \left((n-m) \frac{\pi}{2}\right), \\
\alpha_{m, n}= & -\frac{\left(\zeta_{m}-\zeta_{n}\right)^{2}}{4\left(\sigma_{n}^{2}+\sigma_{m}^{2}\right)},
\end{aligned}
$$

with $\sigma_{0}=\sigma$ and $\zeta_{0}=0$.

The contributions with $n-m \equiv$ odd vanishes, so that Eq. (2.9) can be rewritten as

$$
\begin{aligned}
I(\tau)= & \sqrt{2} \sum_{m=0}^{\infty} \sum_{p=0}^{\infty}(-1)^{p}\left(\pi g_{0}\right)^{2(m+p)} A_{m}^{\star} A_{m+2 p} \\
& \times \sqrt{\frac{\sigma_{m} \sigma_{m+2 p}}{\sigma_{m}^{2}+\sigma_{m+2 p}^{2}}} e^{\alpha_{m, m+2 p}} .
\end{aligned}
$$

The physical meaning of the various terms is fairly transparent, the contributions with $p \neq 0$ are proportional to $e^{-p^{2}(\Delta \tau / \sigma)^{2}}$, they are physically associated to the slippage mechanism and play a minor role with the increasing of the order.

If the pulse effects are not included, namely by setting $\Delta=0$ in Eq. (1.1), we can write the intensity evolution as [8]

\footnotetext{
${ }^{1}$ It should be remembered that the Colson's dimensionless amplitude is linked to the intensity by $|a|^{2}=8 \pi^{2} \frac{I}{I_{s}}$ with $I_{s}$ being the FEL saturation intensity.
}

$$
\begin{aligned}
I(\tau) & =\frac{\left|A_{0}\right|^{2}}{9}\left[3+2 \cosh \left(\frac{N \tau}{\tau_{g}}\right)+4 \cos \left(\frac{\sqrt{3}}{2} \frac{N \tau}{\tau_{g}}\right) \cosh \left(\frac{N \tau}{2 \tau_{g}}\right)\right] \\
\tau_{g} & =\frac{1}{4 \pi \sqrt{3} \rho} .
\end{aligned}
$$

The previous results hold if we assume that the gain coefficient is practically independent of the longitudinal coordinate. The inclusion of the finite electron pulse effects determines some computational problems, but it is instructive to derive the intensity evolution which, for a Gaussian electron bunch with $\operatorname{rms} \sigma_{z}$ and $g_{0}(z)=g_{0} e^{-(1 / 2)\left(z / \sigma_{z}\right)^{2}}$, reads

$$
\begin{aligned}
I(\tau)= & \int_{-\infty}^{+\infty}|a(z, \tau)|^{2} d z \\
= & \sqrt{2} \sum_{m=0}^{\infty} \sum_{n=0}^{\infty}\left(\pi g_{0}\right)^{m+n} A_{n} A \star_{m} \\
& \times \sqrt{\frac{\sigma_{n} \sigma_{m}}{\sigma_{n}^{2}+\sigma_{m}^{2}+d_{n m}}} e^{\alpha_{n, m}} \cos \left((n-m) \frac{\pi}{2}\right), \\
d_{n m}= & 2 \sigma_{n}^{2} \sigma_{m}^{2} \frac{m+n}{\sigma_{z}^{2}}, \\
\alpha_{n, m}= & -\frac{1}{4} \frac{\left(\zeta_{m}-\zeta_{n}\right)^{2}+\frac{2(m+n)}{\sigma_{z}^{2}}\left(\zeta_{m}^{2} \sigma_{n}^{2}+\zeta_{n}^{2} \sigma_{m}^{2}\right)}{\sigma_{n}^{2}+\sigma_{m}^{2}+d_{n m}} .
\end{aligned}
$$

It is worth stressing that the growth rate is strongly reduced for very short bunches.

The first and second moment of the optical field distribution can be computed as (see the Appendix)

$$
\left\langle z(\tau)^{m}\right\rangle=\frac{\left.\int_{-\infty}^{+\infty} z^{m} a(z, \tau)\right|^{2} d z}{\int_{-\infty}^{+\infty}|a(z, \tau)|^{2} d z}, \quad m=1,2, \ldots
$$

and will be used to evaluate the evolution of the centroid and the packet spreading.

In Fig. 1(a) we report the intensity evolution according to Eqs. (2.11) and (2.12); the differences are due to the short bunch effects, which are not contained in Eq. (2.11). The seed amplitude corresponds to an input peak power $P_{0}=900$ W. In Fig. 1(b) we show the intensity evolution (with and without short bunch length effects) obtained by 1D simulation code PROMETEO.

The packet centroid motion $\bar{z}(\tau)=\left\langle z(\tau)^{1}\right\rangle$ is given in Fig. 2 and the rms $\Sigma(\tau)=\sqrt{\left\langle z(\tau)^{2}\right\rangle-\langle z(\tau)\rangle^{2}}$ of the optical pulse is shown in Fig. 3. The moment $\bar{z}(\tau)$ of Eq. (2.13) is relative to a frame moving at the light velocity, its evolution with respect to the e-bunch is obtained by adding the shift $D=\tau \Delta$ [Fig. 2(a)]. The figures are relevant to the case of a short electron bunch and contain the comparison with PROMETEO simulations. As to the centroid shift the comparison exhibits a qualitative agreement only [Figs. 2(a) and 2(c)]. To make analytical and numerical results closer, we have replaced $\Delta$ with $0.6 \Delta$ thus "simulating" a slower drift velocity. Such a reduction 

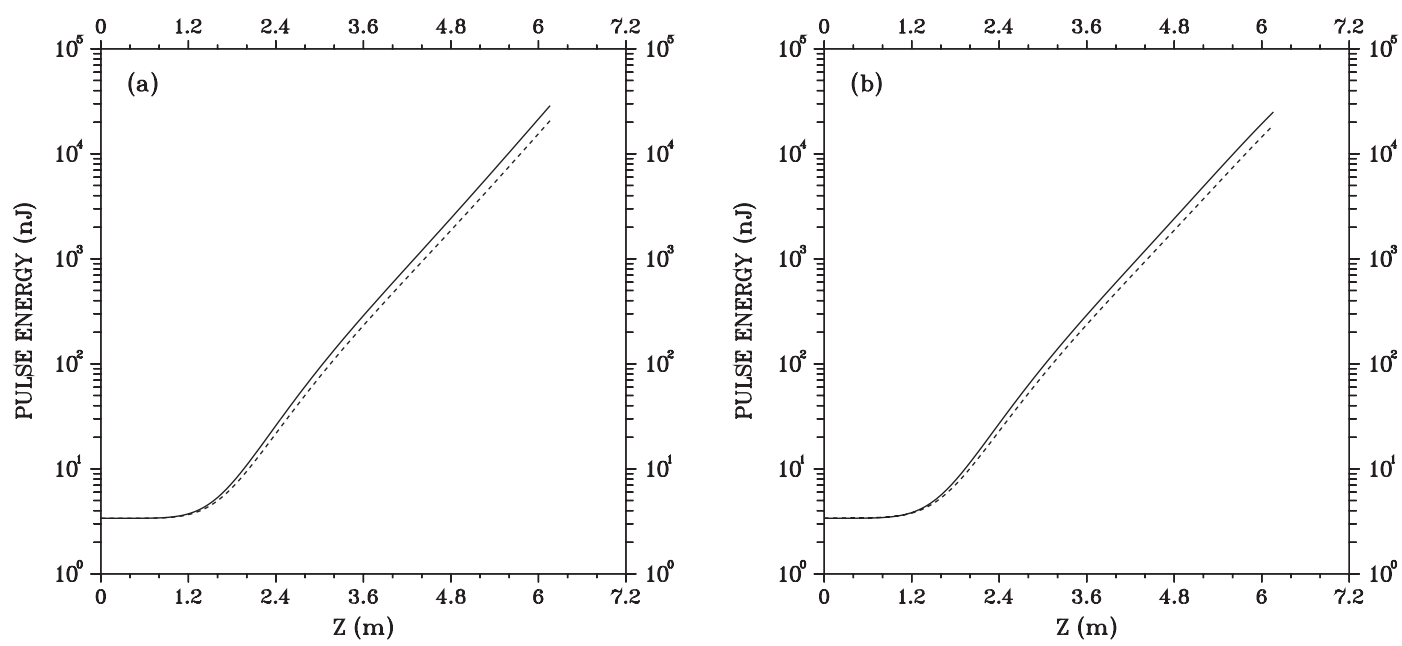

FIG. 1. FEL pulse energy (nJ) vs the undulator coordinate (meters): (a) continuous line from Eq. (2.12), dotted line from Eq. (2.11); (b) PROMETEO simulation: continuous line with short e-bunch, dotted line with continuous electron beam. Parameters: $\lambda_{u}=2.8 \times 10^{-2} \mathrm{~m}, \rho=2.35 \times 10^{-3}, \sigma_{z}=9 \times 10^{-4} \mathrm{~m}, \lambda_{0}=5 \times 10^{-7} \mathrm{~m}, \sigma=4.5 \times 10^{-4} \mathrm{~m}$.
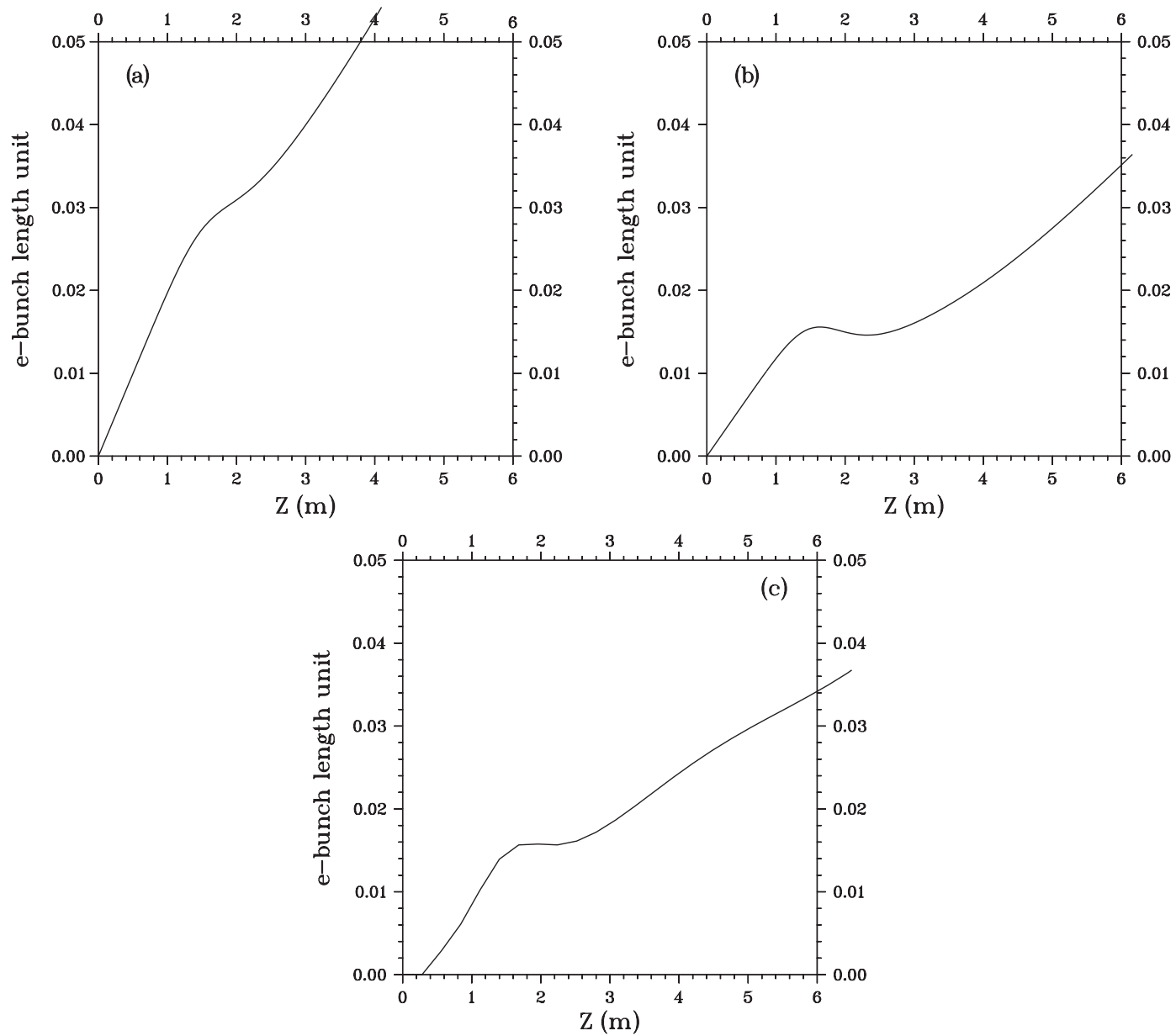

FIG. 2. Centroid position ( $\sigma_{z}$ units) of the optical packet, with respect to the e-bunch, vs the undulator coordinate: (a) from (2.13), $m=1$ and $D=\tau \Delta$; (b) from Eq. (2.13), $m=1$ and $D=0.6 \cdot \tau \Delta$; (c) PROMETEO numerical simulation. Same parameters as Fig. 1 . 

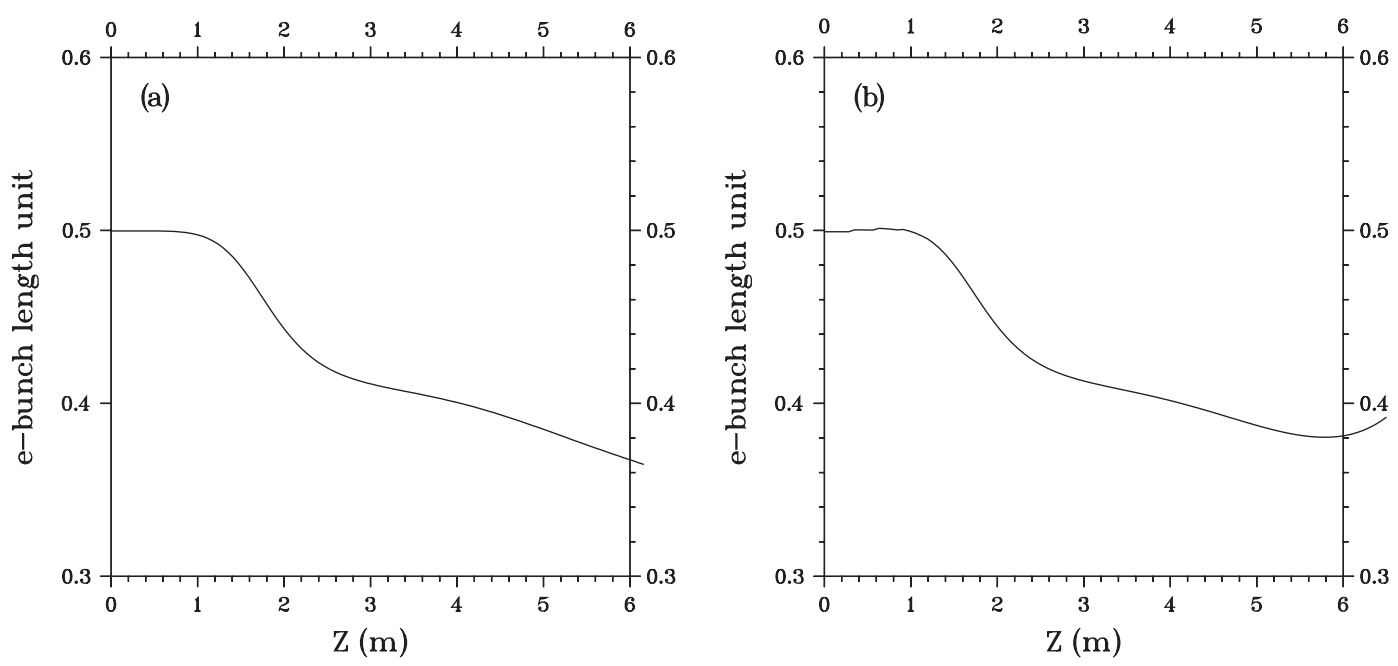

FIG. 3. Packet rms $\Sigma$ ( $\sigma_{z}$ units) vs the undulator coordinate: (a) analytical computation; (b) PROMETEO numerical simulation. Same parameters as Fig. 1.
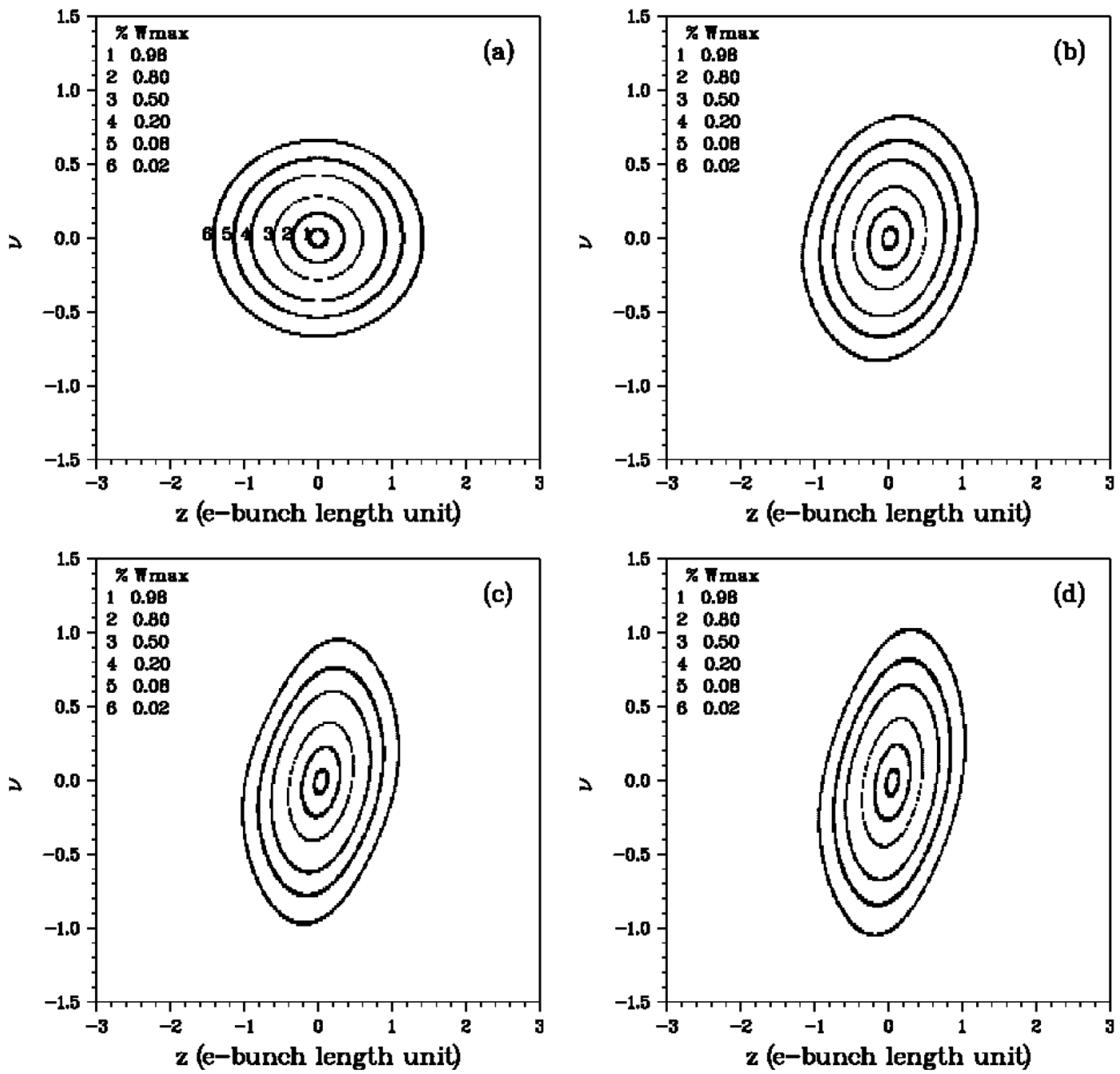

FIG. 4. Wigner phase space contour plots from Eq. (3.7): $W \max =2.0$; same parameters as in Fig. 1 : (a) $Z=0 \mathrm{~m}$; (b) $Z=3.08 \mathrm{~m}$; (c) $Z=6.16 \mathrm{~m}$; (d) $Z=7.98 \mathrm{~m}$ (saturation). 
restores the agreement [see Figs. 2(b) and 2(c)] and can be justified by taking into account that it may be due to intensity dependent effects, not accounted for in (1.1) and to the electron bunch shape effects, not rigorously accounted for in the present analysis in which we have replaced, in the perturbative expansion, $g_{0}$ with $g_{0}(z)$. We have checked the correctness of the second hypothesis repeating the comparison at lower $g_{0}$ values by keeping in the expansion the first term only and by taking into account the dependence of the current on the slippage. The obtained results go in the direction of a better agreement. The check relevant to the intensity dependent effects has been performed by considering an intense input field which has provided more significant differences between the analytical and numerical predictions.

The velocity of the packet calculated as $v_{c}=\frac{d}{d \tau} \bar{z}(\tau)$ from PROMETEO data is consistent with Eqs. (2.2) and (2.3).
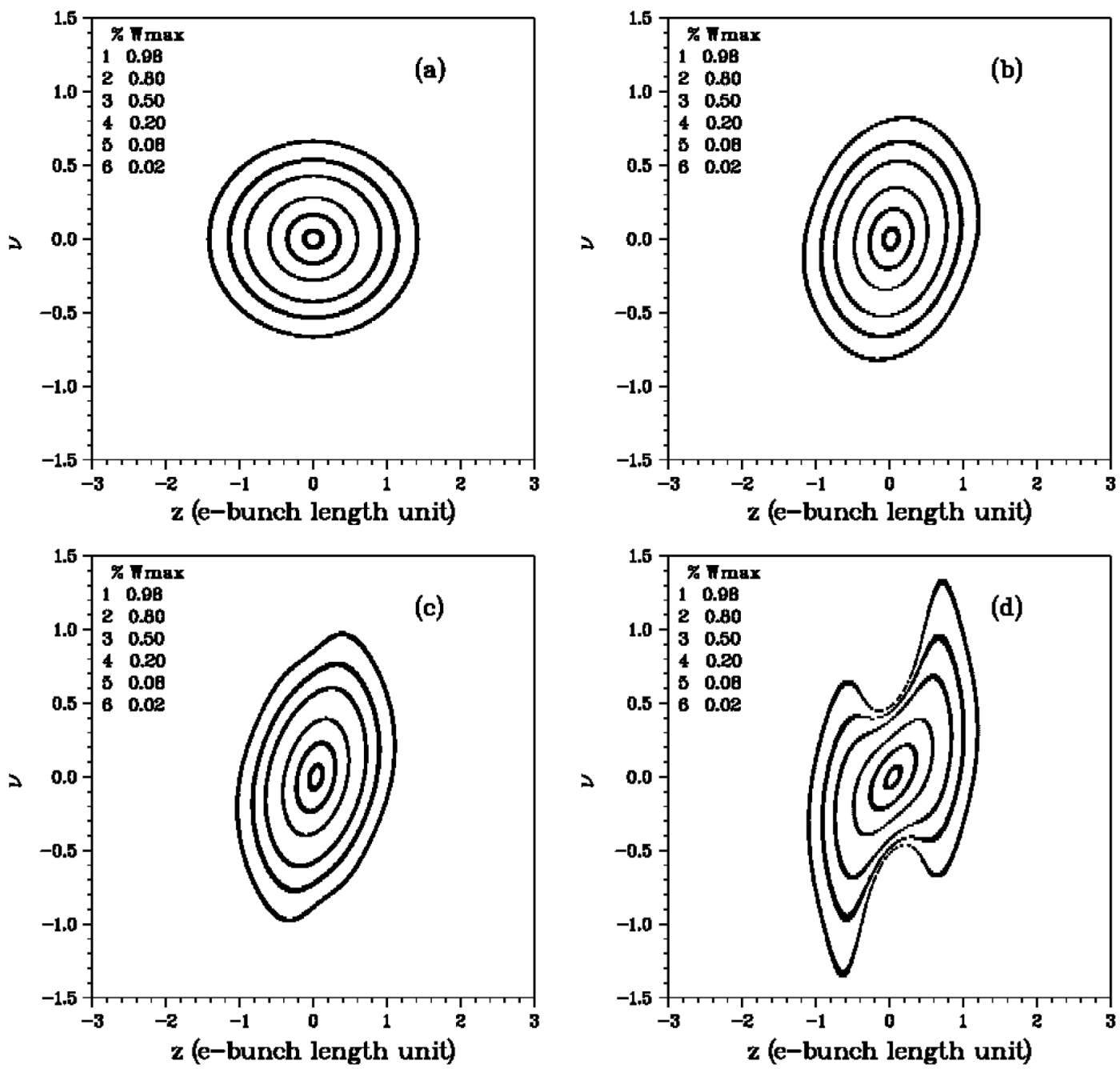

\section{THE FEL WIGNER DISTRIBUTION}

According to the previous discussion, the gain amplitudes $a_{n}(z, \tau)$ have a transparent physical meaning. They can be understood as an optical packet centered at $z_{n}=$ $-\zeta_{n}(\tau)$ and with a packet width provided by $\sigma_{n}(\tau)$. The relevant corresponding spectral properties can be studied by evaluating the Fourier transform of the $a_{n}(z, \tau)$; however, as suggested in Ref. [6], the use of the Wigner distribution may be more convenient.

The Wigner transform of the partial amplitude $a_{n}(z, \tau)$ is

$$
\begin{aligned}
W_{n}(z, \mu, \tau) & =\int_{-\infty}^{\infty} d \eta a_{n}\left(z+\frac{\eta}{2}, \tau\right) a_{n}^{\star}\left(z-\frac{\eta}{2}, \tau\right) e^{-i \eta \mu} \\
& =2\left|A_{n}(\nu, \tau)\right|^{2} e^{-(1 / 2)\left[\left(z+\zeta_{n}\right) / \sigma_{n}\right]^{2}-2 \sigma_{n}^{2}\left(\mu-k_{n}\right)^{2}}
\end{aligned}
$$

where we have defined

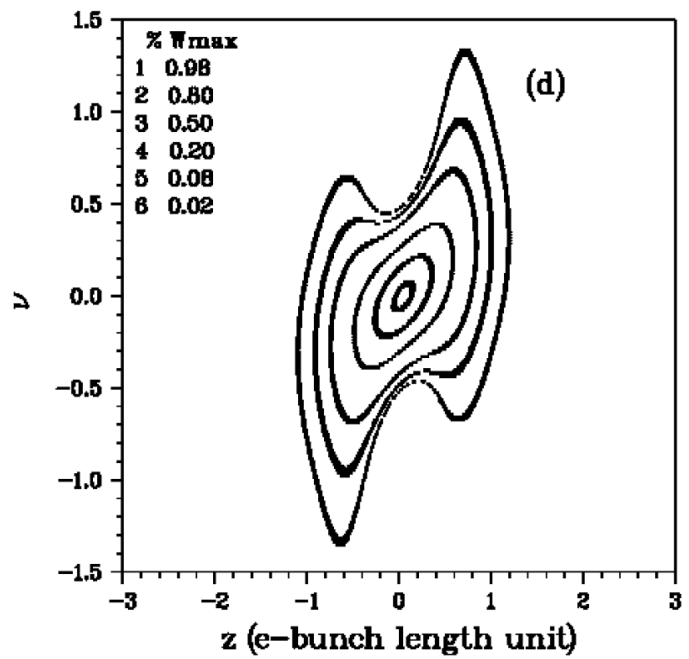

FIG. 5. Wigner phase space contour plots from PROMETEO simulation; same parameters as in Fig. 1: (a) $Z=0 \mathrm{~m}$, $W \max =1.25 \times 10^{-2}$; (b) $Z=3.08 \mathrm{~m}, W \max =3.36 \times 10^{-1}$; (c) $Z=6.16 \mathrm{~m}, W \max =6.96 \times 10^{1}$; (d) $Z=7.98 \mathrm{~m}$, (saturation) $W \max =4.31 \times 10^{2}$. 


$$
\mu=k-k_{0}=-\frac{\nu}{N \lambda_{0}} .
$$

If properly normalized we get from Eq. (3.1) the following rms values:

$$
\begin{gathered}
z_{\mathrm{rms}}=\sigma_{n}(\tau), \quad \mu_{\mathrm{rms}}=\frac{2 \pi}{\lambda_{0}}\left(\frac{\delta \lambda}{\lambda}\right)_{\mathrm{rms}}=\frac{1}{2 \sigma_{n}(\tau)} \\
\delta \lambda=\lambda_{0}-\lambda .
\end{gathered}
$$

The last equation yields for the rms fractional bandwidth

$$
\left(\frac{\delta \lambda}{\lambda}\right)_{\mathrm{rms}}=\frac{1}{4 \pi} \frac{\lambda_{0}}{\sigma_{n}(\tau)} .
$$

The Wigner function of the amplitude $a(z, \tau)$ is

$$
\begin{aligned}
W(z, \mu, \tau) & =\int_{-\infty}^{\infty} d \eta a\left(z+\frac{\eta}{2}, \tau\right) a^{\star}\left(z-\frac{\eta}{2}, \tau\right) e^{-i \eta \mu} \\
& =\sum_{m=0}^{\infty} \sum_{n=0}^{\infty}\left(\pi g_{0}\right)^{m+n} W_{n m}(z, \mu, \tau),
\end{aligned}
$$

where

$W_{n m}(z, \mu, \tau)=\int_{-\infty}^{+\infty} d \eta a_{n}\left(z+\frac{\eta}{2}, \tau\right) a_{m}^{\star}\left(z-\frac{\eta}{2}, \tau\right) e^{-i \eta \mu}$.

If we limit ourselves to the case of zero detuning in Eq. (1.9) and consider $g_{0}(z)$, we obtain for the Wigner function normalized to the intensity $I(\tau)$ :

$$
\begin{aligned}
& W(z, \mu, \tau)=\frac{2 \sqrt{2}}{I(\tau)} \sum_{n=0}^{\infty} \sum_{m=0}^{\infty}\left(\pi g_{0}\right)^{(m+n)} A_{n} A_{m}^{\star}\left(\frac{\sigma_{n} \sigma_{m}}{\sigma_{n}^{2}+\sigma_{m}^{2}+d_{n m}}\right)^{1 / 2} e^{Q_{n m}} \cos \left[(n-m) \frac{\pi}{2}+\mu Z_{n m}\right], \\
& Q_{n m}=-\frac{n+m}{2}\left(\frac{z}{\sigma_{z}}\right)^{2}-\frac{1}{4\left(\sigma_{n}^{2}+\sigma_{m}^{2}+d_{n m}\right)}\left\{\left[\left(z+\zeta_{n}\right)+\left(z+\zeta_{m}\right)\right]^{2}+16 \mu^{2} \sigma_{n}^{2} \sigma_{m}^{2}+R_{n m}\right\}, \\
& Z_{n m}=2 \frac{f_{n m}+2(n-m) \sigma_{n}^{2} \sigma_{m}^{2} z / \sigma_{z}^{2}}{\sigma_{n}^{2}+\sigma_{m}^{2}+d_{n m}}, \quad f_{n m}=\left(z+\zeta_{n}\right) \sigma_{m}^{2}-\left(z+\zeta_{m}\right) \sigma_{n}^{2}, \\
& R_{n m}=d_{n m}\left[\left(\frac{z+\zeta_{n}}{\sigma_{n}}\right)^{2}+\left(\frac{z+\zeta_{m}}{\sigma_{m}}\right)^{2}\right]-\left[2(n-m) \sigma_{n} \sigma_{m} z / \sigma_{z}^{2}\right]^{2}-4 f_{n m}(n-m) z / \sigma_{z}^{2} .
\end{aligned}
$$

The evolution of the relevant phase space contour plots obtained from Eq. (3.7) are reported in Fig. 4 to be compared with the results of PROMETEO simulations reported in Fig. 5.

It is worth noting that a slight correlation in position and frequency occurs, while the power grows along the undulator. The results reported in the previous figures yield just an idea of how the evolution of the Wigner distribution evolves along the undulator; during the linear regime the analytical and the PROMETEO results are in very good agreement which is lost near saturation.

\section{THE EVOLUTION OF THE WIGNER DISTRIBUTION INCLUDING SATURATION}

In the previous sections we have used an analytical procedure useful to study the longitudinal Wigner distribution in the small signal regime. The correctness of the procedure has been benchmarked with PROMETEO, as shown in Figs. 2 and 3. Albeit nonlinear contributions due to saturation can be included using an extension of the logistic function method [7], we use a full numerical procedure since we want to include the effect of the nonlinear harmonic generation.

The evolution of the Wigner distribution will essentially reflect the FEL dynamics; in Fig. 6 we report the evolution of the FEL amplified power of a coherent seed for a set of parameters close to those of the SPARC experiment [9].
In Fig. 6, $k_{3,5}$ denote the knees where the transition to the nonlinear harmonic generation occurs, $p_{1,3,5}$ indicates the first peaks, used as reference for the onset of saturation.

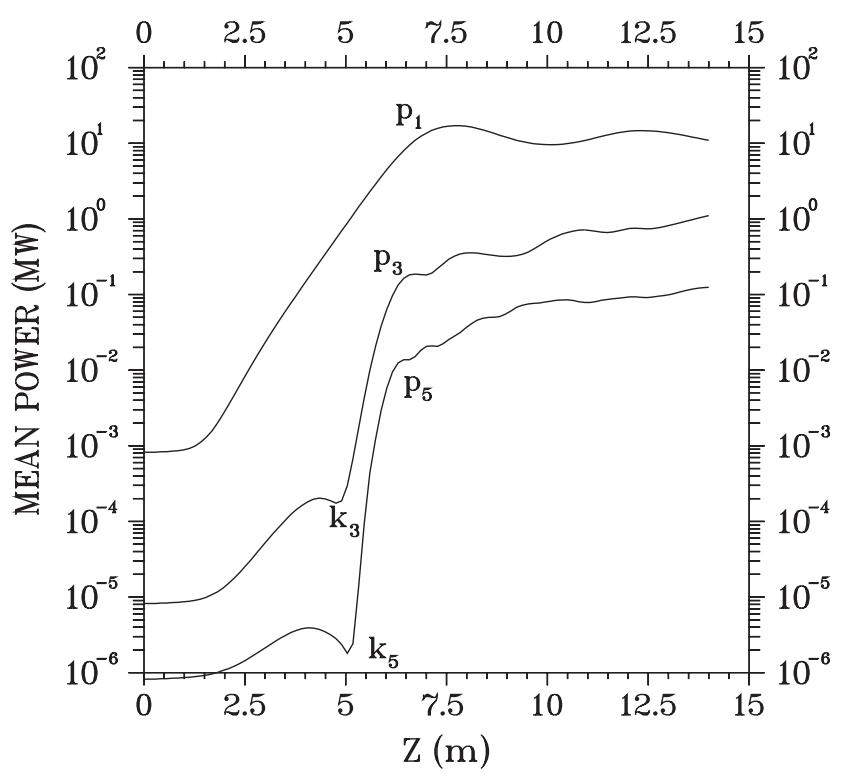

FIG. 6. Evolution of the first three harmonics for SPARC-like parameters: $\lambda_{u}=2.8 \times 10^{-2} \mathrm{~m}, \quad \rho=2.35 \times 10^{-3}, \quad \sigma_{z}=$ $900 \mu \mathrm{m}, \lambda_{0}=5 \times 10^{-7} \mathrm{~m}, \sigma=90 \mu \mathrm{m}, P_{0}=2 \mathrm{KW}$. 
The input seed power has been chosen well below the saturation to avoid any distortion due to early saturation, induced by the seed itself.

We have included the evolution of the power of the first three harmonics and studied the evolution of the Wigner distribution associated with the first, third, and fifth harmonics, along the undulator.

The contour plots of the phase space distribution relevant to the fundamental harmonic are reported in Fig. 7.

The distribution even though initially uncorrelated acquires a small correlation during the linear growth. At the onset of the saturation the phase space curves become distorted (albeit not significantly); a consistent distortion with formation of island and filamentation occurs in the region above saturation where the power undergoes substantial oscillations.

To get a complete understanding of the previous plots, it should be taken into account the evolution of either the square modulus of the optical field pulse and of the spectral distribution, linked to the Wigner distribution by

$$
\begin{aligned}
I(z, \tau) & \propto \int_{-\infty}^{+\infty} W(z, \mu, \tau) d \mu, \\
S(\mu, \tau) & \propto \int_{-\infty}^{+\infty} W(z, \mu, \tau) d z .
\end{aligned}
$$

In Fig. 8 we have reported $I(z, \tau)$ for different positions inside the undulator. A comparison with Fig. 7 shows that the distortion and the formation of island is associated with the distortion of the pulse and the formation of peaks.

The behavior of the phase space evolution of the harmonics is very interesting, because the associated dynamics is more complicated than that of the fundamental. In this case we have essentially three regions of interest (see Fig. 6): (i) the harmonics linear growth and the onset of the bunching, characterized by a "knee" in the power growth curve; (ii) the region of nonlinear coherent generation; and
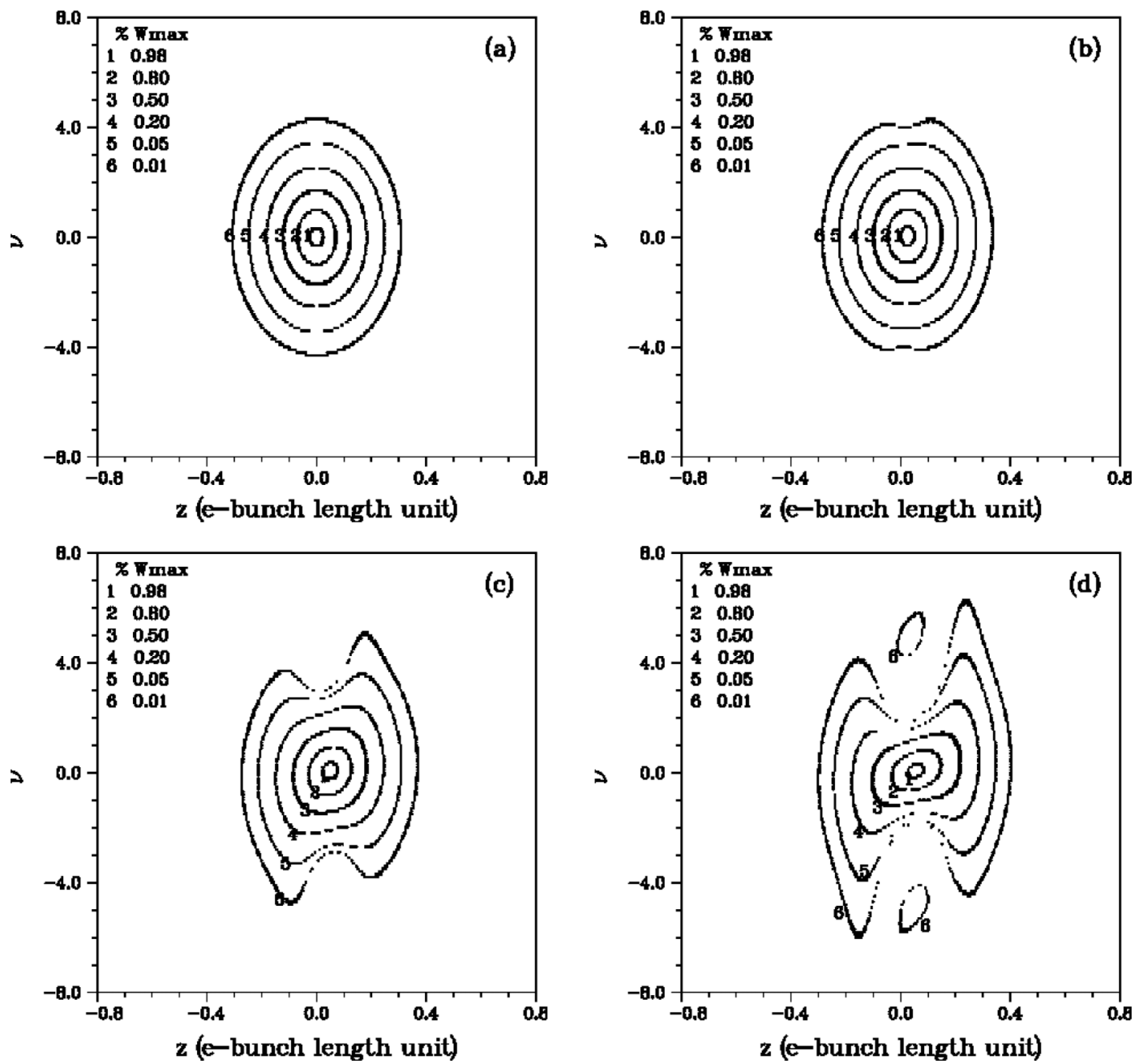

FIG. 7. Phase space distribution of the main harmonic at different positions inside the undulator: (a) $Z=0 \mathrm{~m}, W \mathrm{max}=5.57 \times$ $10^{-3}$; (b) $Z=3.64 \mathrm{~m}, W \max =5.25 \times 10^{-1}$; (c) $Z=6.58 \mathrm{~m}, W \max =6.79 \times 10^{1}$; (d) $Z=7.56 \mathrm{~m}, W \max =1.37 \times 10^{2}$; same parameters as in Fig. 6. 


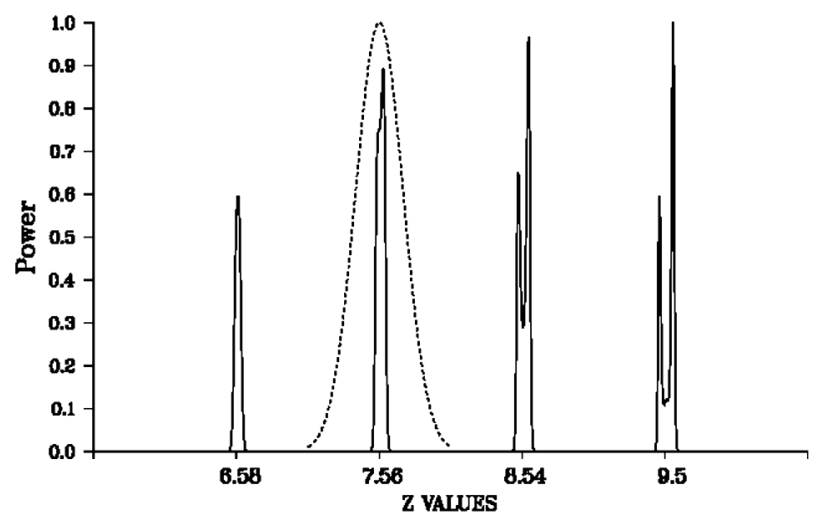

FIG. 8. Pulse shape of the fundamental harmonic at different positions inside the undulator, $1.0=3.39 \times 10^{1}$; same parameters as in Fig. 6. (iii) the saturation. In the first region the harmonic undergoes the same behavior of the fundamental, however when the bunching induced by the fundamental occurs, the phase space is dominated by a significant distortion; in region (b) a kind of recomposition occurs, because the evolution is driven by the fundamental harmonic. Finally, at the saturation the phase space plot becomes distorted and exhibits a kind of chirp, induced by the FEL interaction itself.

The understanding of the evolution of the contour plot of the harmonics (Figs. 9 and 10) can be corroborated by the inspection of Fig. 11 where we have shown the third harmonic pulse shape at different positions inside the undulator (around the knee and at the saturation). Also in this case the phase space distortions are associated with the appearance of peaks in the intensity distribution.
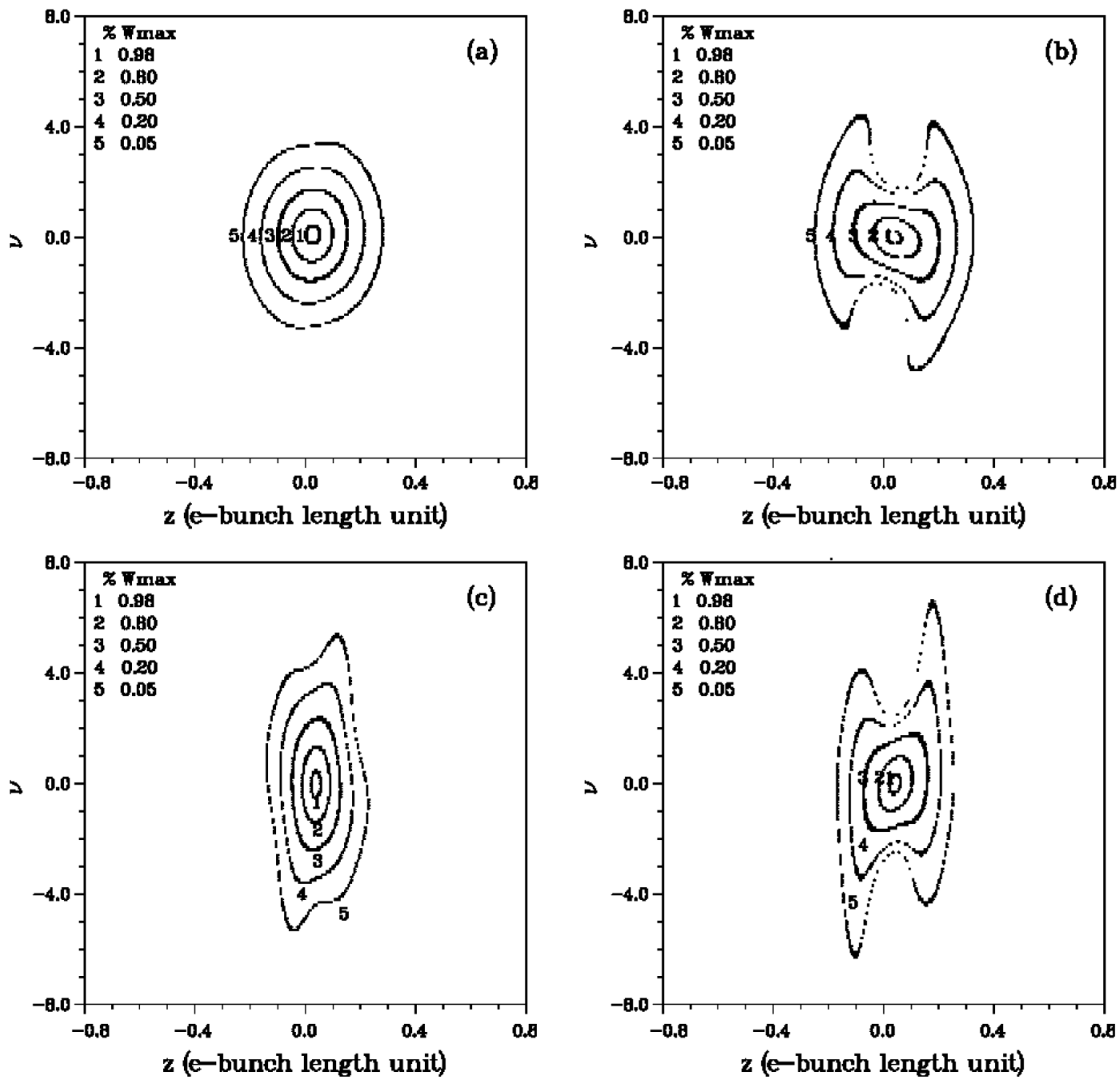

FIG. 9. Phase space contour plot for the third harmonic: (a) linear growth, $W \max =3.28 \times 10^{-4}$; (b) at the knee, $W$ max $=$ $1.35 \times 10^{-3}$; (c) nonlinear harmonic generation region, $W \max =3.02 \times 10^{-1}$; (d) at the occurrence of the first peak, $W$ max $=1.06$; same parameters as in Fig. 6. 

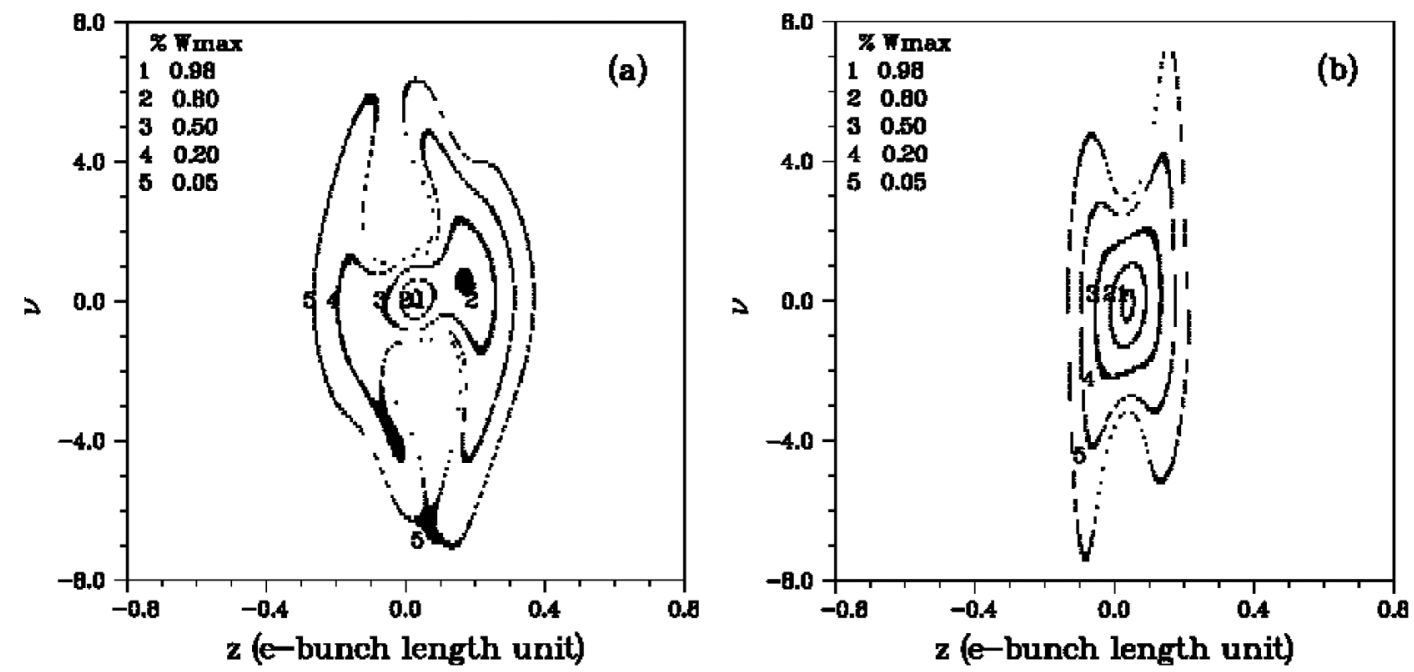

FIG. 10. Fifth harmonic: (a) at the knee, $W \max =1.34 \times 10^{-5}$; (b) at the first peak, $W$ max $=6.39 \times 10^{-2}$; same parameters as in Fig. 6.
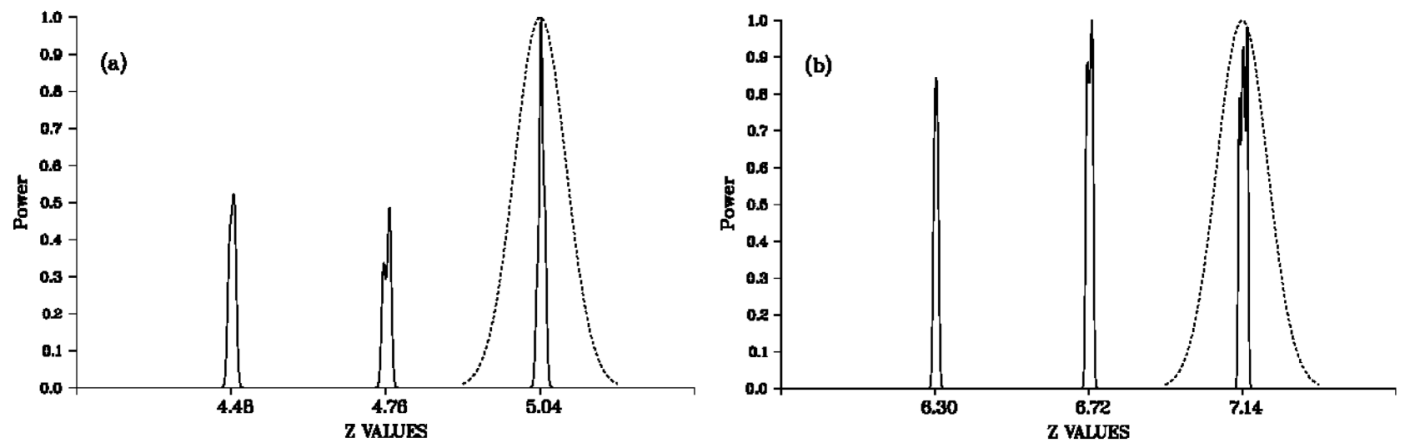

FIG. 11. Third harmonic pulse shape at different positions inside the undulator: (a) around the knee, $1.0=7.71 \times 10^{-4} \mathrm{MW}$; (b) around the saturation, $1.0=3.21 \times 10^{-1} \mathrm{MW}$.

The results of this section provide a quite interesting information on the phase space evolution, which will be further commented on in the next section.

\section{COMPARISON WITH 3D CODES AND FINAL DISCUSSION}

The analysis developed in Secs. II and III is based on the one-dimensional code PROMETEO, in which we added some correcting factors to account for the diffraction contributions. Such a procedure, although satisfactory in reproducing the increase of the gain length due to diffraction, cannot be considered satisfactory as to the details of the evolution of the phase space distribution.

However, since we are considering here the longitudinal phase space only, we expect that therefore the effect of the transverse dynamics come in through an average on the transverse dimensions. ${ }^{2}$

\footnotetext{
${ }^{2}$ The Wigner distribution is therefore defined as $\langle W(x, y, z, \mu ; \tau)\rangle_{x, y}$, where $W(x, y, z, \mu ; \tau)=\int_{-\infty}^{\infty} d \eta a(x, y, z+$ $\left.\frac{\eta}{2}, \tau\right) a^{\star}\left(x, y, z-\frac{\eta}{2}, \tau\right) e^{-i \eta \mu}$ and the average is taken on the transverse coordinates.
}

To better appreciate the effects, we consider the threedimensional generalization of Eq. (1.1):

$$
\begin{aligned}
& -\frac{i}{4} k \nabla_{\perp}^{2} a(x, y, z, \tau)+\partial_{\tau} a(x, y, z, \tau) \\
& =i \pi g_{0}(x, y, z+\Delta \tau) \\
& \quad \times \int_{0}^{\tau} \tau^{\prime} e^{-i \nu \tau^{\prime}} a\left(x, y, z+\Delta \tau^{\prime}, \tau-\tau^{\prime}\right) d \tau^{\prime},
\end{aligned}
$$

which can be treated using an extension of the procedure described in the introductory sections. If we neglect the dependence of the gain coefficient on the transverse coordinates we can write Eq. (5.1) in the form [1]

$$
\begin{aligned}
\partial_{\tau} \tilde{a}(x, y, z, \tau) & =i \pi g_{0} \int_{0}^{\tau} \tau^{\prime} e^{-i \hat{\nu} \tau^{\prime}} \tilde{a}\left(x, y, z, \tau-\tau^{\prime}\right) d \tau^{\prime}, \\
\tilde{a}(x, y, z, \tau) & =e^{-(i \nu k / 4) \nabla_{\perp}^{2}} a(x, y, z, \tau), \\
\hat{\nu} & =\nu+i \Delta \partial_{z}-\frac{k}{4} \nabla_{\perp}^{2} .
\end{aligned}
$$

The above equation is formally equivalent to that reported in Eq. (1.3) and the relevant solutions can be obtained in complete analogy with the one-dimensional case, 
at the cost of a few complications, due to the computation of the action of the transverse Laplacian $\nabla_{\perp}^{2}$ on the field amplitude [10].

We do not report the inclusion of transverse effects in terms of this procedure and make a comparison between numerical full numerical codes.

We have compared the numerical results from PROMETEO with the code GENESIS including threedimensional effects. The first check has been done by confronting the growth of the amplified power with the two codes as shown in Fig. 12.

The comparison is satisfactory even though GENESIS includes the effect of e-beam propagation through the transport channel and the drift sections between the undulators.

The longitudinal phase space plots obtained with the two codes are reported in Fig. 13. We have compared the region near the saturation, where significant distortions occur. The plots have been confronted at equivalent undulator coordinate, namely by subtracting the drift lengths in the case of GENESIS.

The Wigner distribution phase space plots predicted by the different numerical procedures, Fig. 13, show a satisfactory agreement.

The differences may be due to manifold reasons, as, e.g., the smoothing due to the diffraction or it can even be associated with the averaging procedure on the transverse coordinate, which induces a nonphysical smoothing.

The longitudinal phase space evolution of the FEL radiation depends on the FEL dynamics, but also on the characteristics of the input seed. In particular, if it is characterized by a chirp

$$
a_{0}(z)=\frac{A_{0}}{\sqrt[4]{2 \pi \sigma^{2}}} e^{-z^{2} / 4 \sigma^{2}} e^{i \alpha\left(z^{2} / \sigma^{2}\right)}
$$

the initial distribution we are referring to is

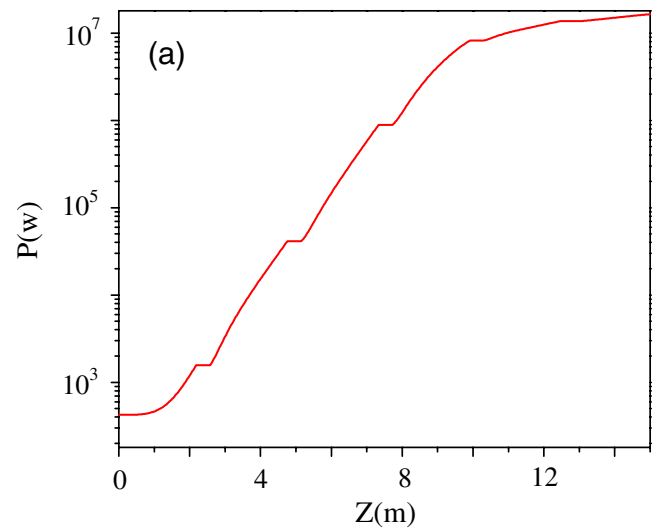

$$
\begin{gathered}
W(z, \mu, 0) \propto e^{-\left(A z^{2}+2 B z \mu+C \mu^{2}\right)}, \quad A=\frac{1+16 \alpha^{2}}{2 \sigma^{2}}, \\
B=-4 \alpha, \quad C=2 \sigma^{2},
\end{gathered}
$$

where $\alpha$ is the energy phase correlation parameter, the coefficients $A, B$, and $C$ are Twiss-like parameters satisfying the normalization condition $A C-B^{2}=1$. The sign of $\alpha$ determines the orientation of the phase space ellipse; for positive values the ellipse major axis forms with the $z$ axis an angle larger than $\frac{\pi}{2}$.

In Fig. 14 we show the phase space distribution of the fundamental harmonic taken at the first peak, for different values of the correlation parameter.

The initial slope of the contour plot distributions, determined by the correlation parameter, does not appear modified by the interaction itself. On the other hand, if the electron bunch too exhibits an energy phase correlation, the associated evolution of the field phase space distribution contains some additional elements which are worth pointing out. To make the effect of the electron chirping more significant on the dynamics of the amplification evolution, we have assumed that the input seed longitudinally overlaps the electron bunch (both Gaussian pulses with the same rms). The evolution of the Wigner phase space plots are characterized by a rotation and by a shift (depending on the sign of the correlation parameter) of the center of the spectral distribution (see Fig. 15). We have assumed that, initially, the e-beam is characterized by a phase longitudinal distribution, analogous to that of the input field given in Eq. (5.4), with $\alpha_{c}$ denoting the energy phase correlation:

$p(\epsilon, z)=\frac{1}{2 \pi \Sigma} e^{-(1 / 2 \Sigma)\left(\gamma_{c} z^{2}+2 \alpha_{c} z \epsilon+\beta_{c} \epsilon^{2}\right)}, \quad \epsilon=\frac{\gamma-\gamma_{0}}{\gamma_{0}}$,

where $\Sigma$ is the longitudinal emittance.

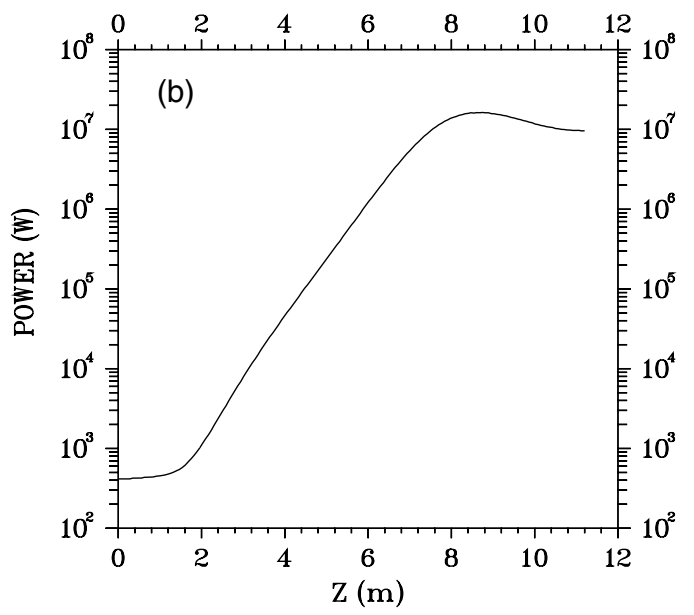

FIG. 12. Main harmonic power evolution: (a) GENESIS 3D simulation; (b) PROMETEO 1D simulation. 

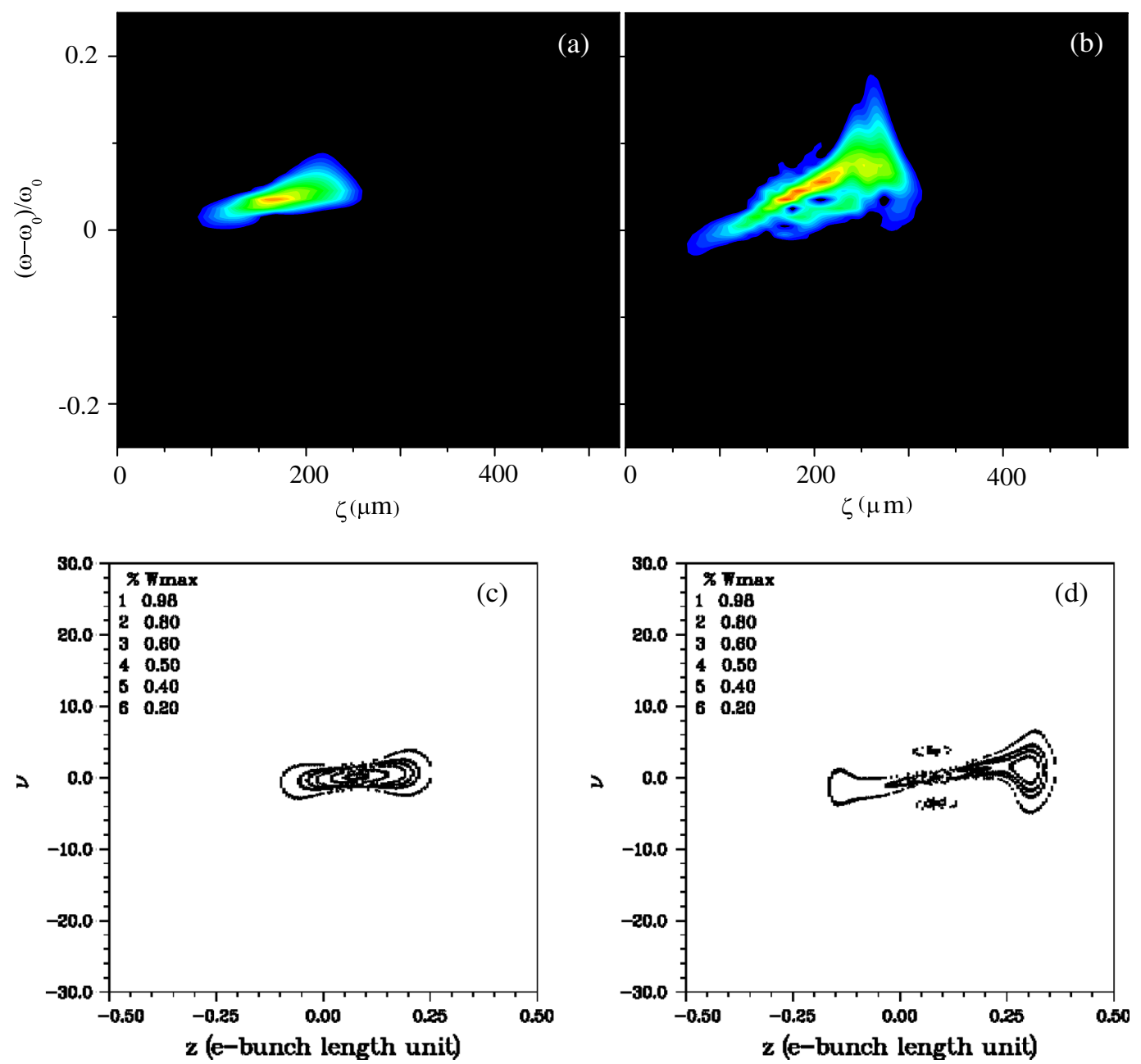

FIG. 13. Wigner distribution phase space plots; GENESIS 3D simulation: (a) $Z=10.68 \mathrm{~m}$ and (b) $Z=13.35 \mathrm{~m}$; PROMETEO $1 \mathrm{D}$ simulation: (c) $Z=8.82 \mathrm{~m}$ (saturation), $W \max =9.17 \times 10$ and (d) $Z=11.06 \mathrm{~m}, W \max =6.18 \times 10$.

The comparison of Fig. 15(a) with Fig. 14(c) seems to suggest that the electron bunch chirping, with opposite sign to that of the radiation, determines, after the interaction, a noncorrelated distribution. This is however just a qualitative statement deserving further analysis.

In this paper we have presented a fairly comprehensive analysis of a high gain FEL amplifier dynamics, through the properties of the Wigner transform of the associated field.

The physical content of our discussion has been mainly focused on the evolution of the phase space plots, which have revealed interesting elements of discussion. One of these, which resulted rather surprising, is the fact that the FEL interaction does not induce any significant energy phase correlation (see, e.g., Fig. 7). It is evident that the interpretation of the Wigner distribution evolution is hampered by the fact we are not considering a "conservative" dynamics, the optical field is going through an amplification process exhibiting a strong nonlinearity, which induces a significant distortion of the initial Gaussian distribution, thereby inducing higher order moments and the description in terms of Twiss-like parameters is inadequate. However, if we limit ourselves to the linear regime (before the saturation), we can still interpret the evolution of the longitudinal phase space in terms of a distribution given in Eq. (5.4).

A quantitative analysis of the problem under study demands for an appropriate definition of the parameters we have introduced.

The linear chirp parameter is linked to the phase of the associated electric field by the relation

$$
\chi=-\left.\frac{d^{2}}{d t} \varphi\right|_{t=0}
$$

We will define a Gaussian chirped pulse with group delay dispersion (GDD) in the spectral domain as

$$
\tilde{E}(\omega) \propto e^{-\left(T_{G 0} / 2\right)^{2}\left(\omega-\omega_{0}\right)^{2}} e^{i(g / 2)\left(\omega-\omega_{0}\right)^{2}},
$$



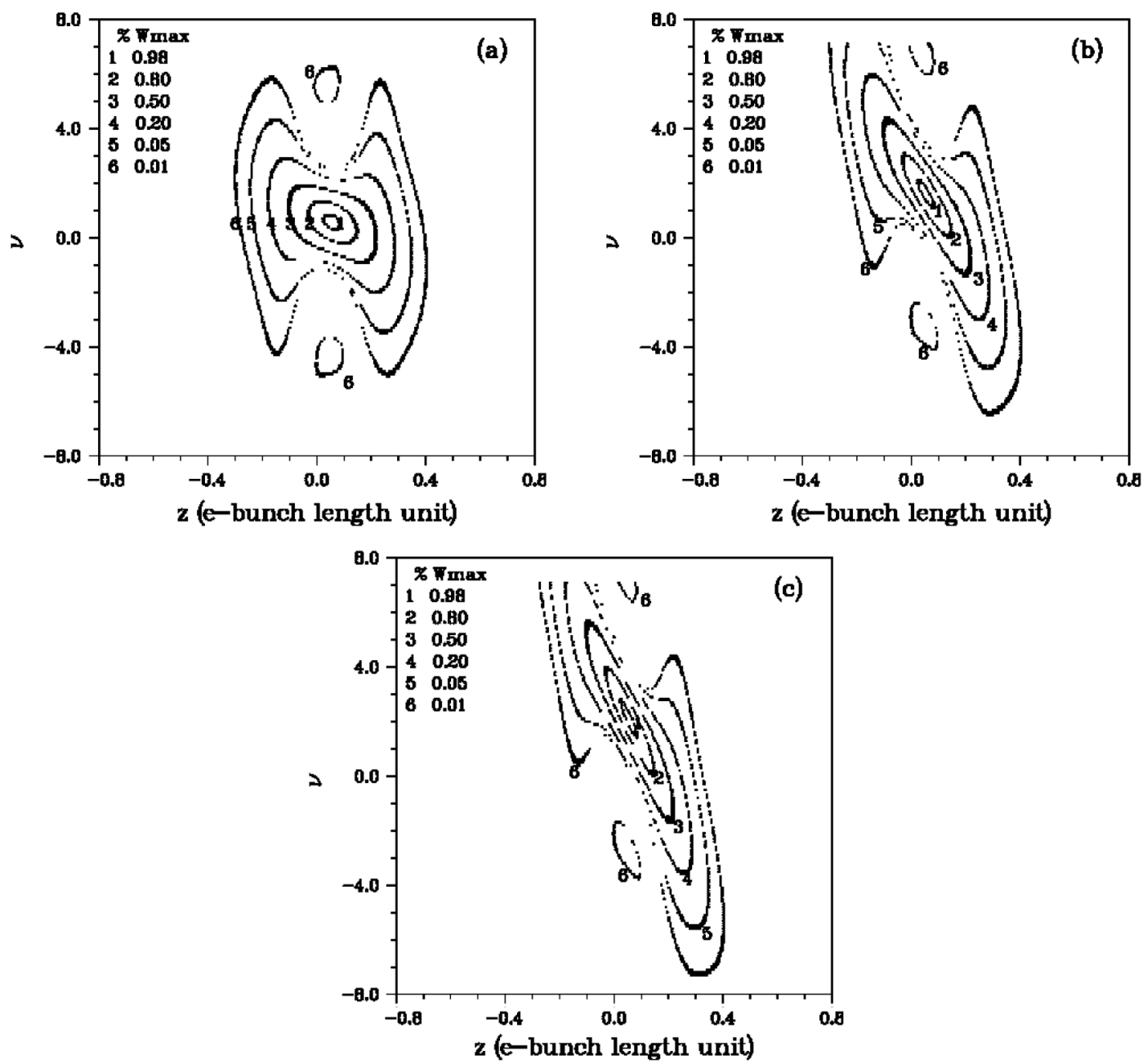

FIG. 14. Phase space plot at the first peak of the fundamental for different values of the correlation parameter $\alpha, W$ max $=$ $1.37 \times 10^{2}$ : (a) $\alpha=0.1$, (b) $\alpha=0.3$, (c) $\alpha=0.4$; same parameters as in Fig. 6 .
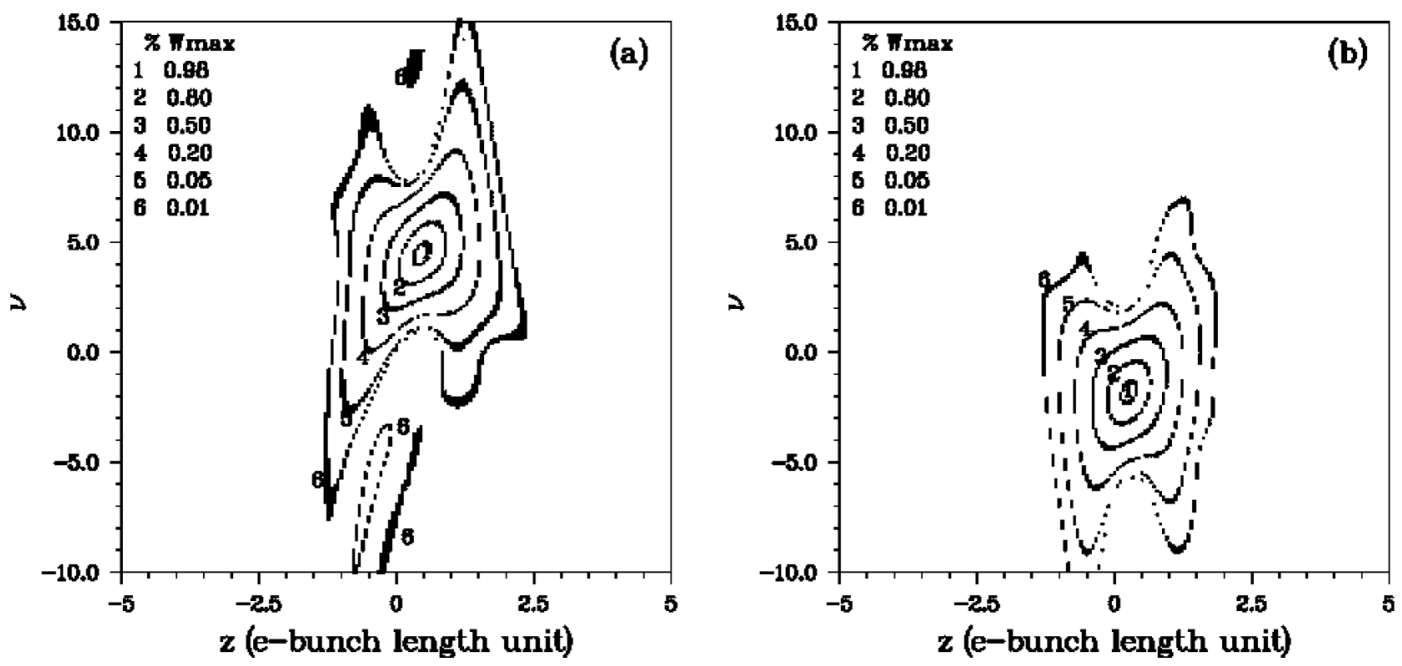

FIG. 15. Phase space plot at the first peak of the fundamental for different values of the correlation parameter $\alpha_{c}$ : (a) $\alpha_{c}=-4$, $W \max =5.47 \times 10$, (b) $\alpha_{c}=4, W \max =4.59 \times 10$; simulation parameters: $\lambda_{u}=2.8 \times 10^{-2} \mathrm{~m}, \rho=2.35 \times 10^{-3}, \sigma_{z}=\sigma=$ $9 \times 10^{-5} \mathrm{~m}, \lambda_{0}=5 \times 10^{-7} \mathrm{~m}, \alpha=0.4$. 

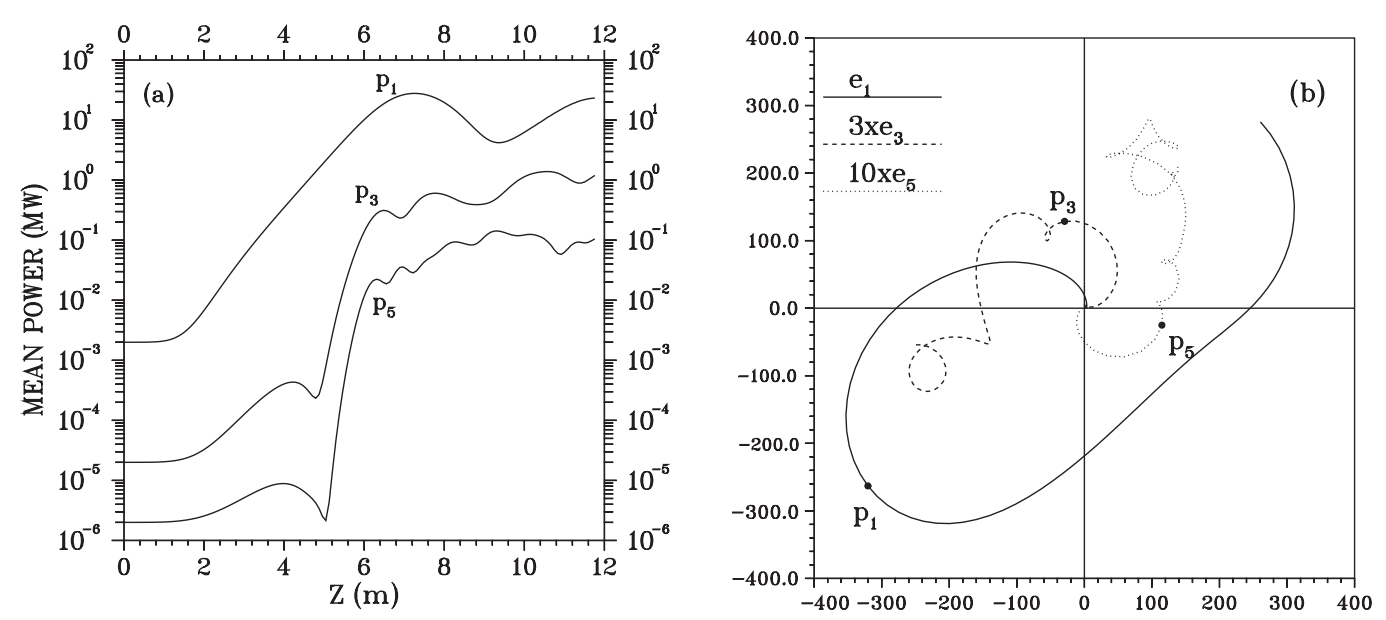

FIG. 16. (a) Evolution of the fundamental, third and fifth harmonics vs the undulator length. (b) Imaginary and real part of the electric fields associated with the power in Fig. (a); the dots mark the values at first maxima. Simulation parameters: $\lambda_{u}=$ $2.8 \times 10^{-2} \mathrm{~m}, \rho=2.35 \times 10^{-3}, \lambda_{0}=5 \times 10^{-7} \mathrm{~m}, P_{0}=2 \mathrm{KW}$.

where $g$ is the GDD parameter and $T_{G 0}=\frac{T_{P}}{\sqrt{2 \ln (2)}}$ with $T_{P}$ being the chirp-free pulse duration in full width at half maximum. By Fourier transform of the field in Eq. (5.3), we obtain the field in the time domain and we can evaluate the chirp as

$$
\chi=\frac{4 g}{T_{G 0}^{4}+4 g^{2}} .
$$

The associated Wigner transform yields

$$
W(t, \omega)=e^{-\left(1 / 2 T_{G 0}^{2}\right)\left[4 t^{2}-8 g \omega t+\left(T_{G 0}^{4}+4 g^{2}\right) \omega^{2}\right]} .
$$

Therefore, by making a comparison with the definition in terms of Twiss parameters, we obtain

$$
\gamma \rightarrow \frac{2}{T_{G 0}^{2}}, \quad|\alpha| \rightarrow \frac{2 g}{T_{G 0}^{2}}, \quad \beta \rightarrow \frac{T_{G 0}^{4}+4 g^{2}}{2 T_{G 0}^{2}} .
$$

The optical pulse length will be therefore proportional to

$$
\sqrt{\beta}=\frac{T_{G 0}}{\sqrt{2}} \sqrt{1+4\left(\frac{g}{T_{G 0}^{2}}\right)^{2}} .
$$

Finally, by making a comparison with Eq. (1.9) we infer that the chirping associated to this field amplitude is provided by

$$
g \propto\left(\frac{\Delta \tau}{\gamma_{n}}\right) .
$$

The effect of the dispersion does not appear significant during the linear regime, but becomes more and more important while the field grows.

An alternative picture of the evolution of FEL can be provided by that of the complex electric field. In Fig. 16(b) we have reported in an Argand-Gauss plane the imaginary vs the real part of the FEL normalized electric field ${ }^{3}$ of the fundamental and of the higher order harmonics, associated with the evolution of the relevant power reported in Fig. 16(a). The plot in (b) exhibits a "gentle" behavior of the electric field of the fundamental, characterized by a rotation in the complex plane along with an increase of its modulus. The higher order harmonics are characterized by a bizarre behavior ${ }^{4}$ after the first maximum (the dots are the markers denoting the complex field of the fundamental at the first maximum, while that of the harmonics just before the knee). The field phases grow almost linearly with the undulator coordinate, before the relevant intensities have reached the marked points. The transition to the nonlinear harmonic generation is characterized by an involved behavior.

Albeit referring to the case in which the pulse propagation effects have not been included, the plots of Fig. 16 yield an idea of the usefulness of the Argand-Gauss plot of the FEL electric field, which can complement the information obtained from the analysis of the Wigner distribution evolution, as it will be shown in a forthcoming paper.

\section{APPENDIX}

In Sec. II we have reported the partial amplitudes and the intensity growth, calculated at zero detuning. The reason of such a choice is merely due to the fact that the analytical expressions are easy to manage and more transparent from the physical point of view.

\footnotetext{
${ }^{3} \mathrm{By}$ normalized electric field we mean $e_{s}\left[\mathrm{~m}^{-1}\right]=$ $3.79834 \sqrt{I\left[\frac{\mathrm{MW}}{\mathrm{cm}^{2}}\right]} e^{-i \varphi_{s}}$ where $I$ is the field intensity and $\varphi_{s}$ its phase.

${ }^{4}$ Probably due to the fact that the vectors associated with the various harmonics evolve in different planes and we should visualize the relevant plot on a three-dimensional surface.
} 
We report below the more general cases; the relevant computation is rather cumbersome, but straightforward.

In the following we omit the $\nu, \tau$ dependence of the $A_{n}, \sigma_{n}, \zeta_{n}$ :

Total field square amplitude:

$$
\begin{gathered}
|a(z, \tau)|^{2}=\frac{1}{\sqrt{2 \pi}} \sum_{m=0}^{\infty} \sum_{n=0}^{\infty}\left(\pi g_{0}\right)^{m+n} \frac{A_{n} A_{m}^{\star}}{\sqrt{\sigma_{n} \sigma_{m}}} e^{-1 / 4\left[\left(z+\zeta_{n}\right)^{2} / \sigma_{n}^{2}+\left(z+\zeta_{m}\right)^{2} / \sigma_{n}^{2}\right]} \cos \left(\Phi_{n, m}\right), \quad \Phi_{n, m}=\left(k_{n}-k_{m}\right) z+\Delta_{n, m}, \\
\Delta_{n, m}=(n-m) \frac{\pi}{2}+\left(\omega_{m}-\omega_{n}\right) \tau+k_{n} \zeta_{n}-k_{m} \zeta_{m} .
\end{gathered}
$$

Field intensity:

$$
\begin{aligned}
I(\tau) & =\int_{-\infty}^{+\infty}|a(z, \tau)|^{2} d z=\sqrt{2} \sum_{m=0}^{\infty} \sum_{n=0}^{\infty}\left(\pi g_{0}\right)^{n+m} A_{n} A_{m}^{\star} \sqrt{\frac{\sigma_{n} \sigma_{m}}{\sigma_{n}^{2}+\sigma_{m}^{2}}} e^{\alpha_{n, m}} \cos \left(\beta_{n, m}\right), \\
\alpha_{n, m} & =-\frac{\sigma_{n}^{2} \sigma_{m}^{2}}{4\left(\sigma_{n}^{2}+\sigma_{m}^{2}\right)}\left[\frac{\left(\zeta_{n}-\zeta_{m}\right)^{2}}{\sigma_{n}^{2} \sigma_{m}^{2}}+4\left(k_{n}-k_{m}\right)^{2}\right], \quad \beta_{n, m}=\Delta_{n, m}-\left(k_{n}-k_{m}\right) \frac{\zeta_{m} \sigma_{n}^{2}+\zeta_{n} \sigma_{m}^{2}}{\sigma_{n}^{2}+\sigma_{m}^{2}}
\end{aligned}
$$

First and second moments:

$$
\begin{aligned}
\langle z(\tau)\rangle & =\frac{\int_{-\infty}^{+\infty} z|a(z, \tau)|^{2} d z}{\int_{-\infty}^{+\infty}|a(z, \tau)|^{2} d z}=-\frac{\sqrt{2}}{I(\tau)} \sum_{m=0}^{\infty} \sum_{n=0}^{\infty}\left(\pi g_{0}\right)^{n+m} A_{n} A_{m}^{\star} \sqrt{\frac{\sigma_{n} \sigma_{m}}{\sigma_{n}^{2}+\sigma_{m}^{2}}} e^{\alpha_{n, m}} \Lambda_{n, m}, \\
\Lambda_{n, m} & =\frac{\left(\zeta_{m} \sigma_{n}^{2}+\zeta_{n} \sigma_{m}^{2}\right) \cos \left(\beta_{n, m}\right)+2\left(k_{n}-k_{m}\right)\left(\sigma_{n} \sigma_{m}\right)^{2} \sin \left(\beta_{n, m}\right)}{\sigma_{n}^{2}+\sigma_{m}^{2}}
\end{aligned}
$$

and

$$
\begin{aligned}
\left\langle z(\tau)^{2}\right\rangle & =\frac{\sqrt{2}}{I(\tau)} \sum_{m=0}^{\infty} \sum_{n=0}^{\infty}\left(\pi g_{0}\right)^{n+m} A_{n} A_{m}^{\star} \sqrt{\frac{\sigma_{n} \sigma_{m}}{\sigma_{n^{2}}+\sigma_{m^{2}}}} \Gamma_{n, m}, \quad \Gamma_{n, m}=e^{\alpha_{n, m}}\left(\frac{\sigma_{n} \sigma_{m}}{\sigma_{n}^{2}+\sigma_{m}^{2}}\right)^{2}\left[D_{n, m} \cos \left(\beta_{n, m}\right)+E_{n, m} \sin \left(\beta_{n, m}\right)\right], \\
D_{n, m} & =2\left(\sigma_{n}^{2}+\sigma_{m}^{2}\right)+\left(\frac{\zeta_{m} \sigma_{n}^{2}+\zeta_{n} \sigma_{m}^{2}}{\sigma_{n} \sigma_{m}}\right)^{2}-4\left(\sigma_{n} \sigma_{m}\right)\left(k_{n}-k_{m}\right)^{2}, \quad E_{n, m}=4\left(k_{n}-k_{m}\right)\left(\zeta_{m} \sigma_{n}^{2}+\zeta_{n} \sigma_{m}^{2}\right) .
\end{aligned}
$$

[1] G. Dattoli, A. Renieri, and A. Torre, Lectures on Free Electron Lasers and on Related Topics (World Scientific, Singapore, 1990).

[2] W. B. Colson, in Laser Handbook, edited by W. B. Colson, C. Pellegrini, and A. Renieri (North-Holland, Amsterdam, 1990), Vol. V.

[3] R. Bonifacio, C. Pellegrini, and L. M. Narducci, Opt. Commun. 50, 373 (1984).

[4] G. Dattoli, L. Giannessi, A. Torre, and A. Segreto, J. Appl. Phys. 79, 6729 (1996).

[5] H. M. Srivastava and L. Manocha, A Treatise on Generating Functions (John Wiley \& Sons, New York, 1989).

[6] S. Krinsky and Z. Huang, Phys. Rev. ST Accel. Beams 6, 050702 (2003); J. Wu, J. B. Murphy, P. J. Emma, X. Wang, T. Watanabe, and X. Zhong, J. Opt. Soc. Am. B 24, 484 (2007); A. Lutman, G. Penco, P. Craievich, and J. Wu, J. Phys. A 42, 085405 (2009).
[7] R. Bonifacio, L. De Salvo, P. Pierini, N. Piovella, and C. Pellegrini, Phys. Rev. Lett. 73, 70 (1994); J. B. Murphy, J. Wu, X.J. Wang, and T. Watanabe, Reports No. BNL-75807- 2006-JA and No. SLAC-PUB11852, 2006.

[8] I. B. Bernstein and J. L. Hirshfield, Phys. Rev. A 20, 1661 (1979); E. L. Saldin, E. V. Schneidmiller, and M. V. Yurkov, The Physics of Free Electron Lasers (Springer, Berlin Heidelberg, 2000); G. Dattoli and P. L. Ottaviani, Opt. Commun. 204, 283 (2002); G. Dattoli, P. L. Ottaviani, and S. Pagnutti, ENEA Frascati Internal Report No. RT/ 2007/40/FIM.

[9] L. Giannessi et al., Phys. Rev. ST Accel. Beams 14, 060712 (2011).

[10] G. Dattoli, A. Dipace, E. Sabia, A. Torre, G. K. Voykov, and M. Carpanese, J. Appl. Phys. 80, 6589 (1996). 